\title{
Numerical analysis on load-bearing capacity and damage of double scarf adhesive joints subjected to combined loadings of tension and bending
}

\author{
Lijuan Liao $^{\mathrm{a}, *}$, Toshiyuki Sawa ${ }^{\mathrm{b}}$, Chenguang Huang ${ }^{\mathrm{a}}$ \\ ${ }^{a}$ Key Laboratory for Mechanics in Fluid Solid Coupling Systems, Institute of Mechanics, Chinese Academy of Sciences, Beijing 100190, \\ People's Republic of China \\ ${ }^{\mathrm{b}}$ Graduate School of Engineering, Hiroshima University, 1-4, Kagamiyama, Higashihiroshima, Hiroshima 739-8527, Japan
}

\section{A R T I C L E I N F O}

Available online 8 January 2014

Keywords:

Double scarf adhesive joint (DSJ)

Cohesive zone model (CZM)

Damage

Failure energy

Mixed mode

\begin{abstract}
A B S T R A C T
The load-bearing capacity and the damage level of the double scarf joint (DSJ) under combined loadings of tension and bending were investigated numerically, which takes into account the effects of scarf angle and adhesive type. A finite element method (FEM), which includes a mixed-mode cohesive zone model (CZM) with a bilinear shape, was employed to govern the interface separation behaviors. At the point corresponding to the ultimate loading, it was observed that the interface damage level of DSJ with the ductile adhesive is higher and more uniform than that of the joint with the brittle one. More than that, the numerical results illustrated that the failure of DSJ is controlled not only by the ultimate loading, but also by the applied displacement until complete failure. Therefore, the failure energy, which is defined as the integral of the loading with respect to the displacement, was adopted to estimate the joint performance. Subsequently, the numerical results showed that the failure energy of the joint with the ductile adhesive is higher than that of the joint with the brittle one. Furthermore, all the discussed characteristic parameters of a DSJ with a given adhesive, including ultimate loading, the von-Mises equivalent stress and interface damage level corresponding to the ultimate loading, and the failure energy, were inversely proportional to the scarf angle. Finally, through comparing with the existing experimental measurements, the adoptive method was validated.
\end{abstract}

(c) 2014 Elsevier Ltd. All rights reserved.

\section{Introduction}

Adhesive joints, with many advantages over conventional mechanical fastening techniques, are an ideal joining method of lightweight and high strength. Strength estimation and failure mechanism examination are crucial to widen the technological applications of adhesive joints subjected to external applied loadings, especially in aerospace and automotive industries. Among all types of adhesive joints, the scarf adhesive joint is commonly adopted to join fiber reinforced laminate composite elements and components for more uniform stress distributions [1-3]. The examinations concerning the effects of the scarf angle on the joint performance under uniaxial tensile loading have been carried out in many studies [2,4-6], which show that the failure loads increase as the characteristic angle decreases. Furthermore, the double scarf adhesive joint (DSJ), with the similar advantages as the single scarf adhesive joint (SSJ), is also used widely in mechanical industries. The obvious

\footnotetext{
*Corresponding author. Tel.: +8610 8254 4291; fax: +8610 82544256.

E-mail address: liaohuanxin@hotmail.com (L. Liao).
}

characteristic of the DSJ is the geometric configuration, which has acute and obtuse angles at the ends of the substrates and the top of the double scarf, respectively [3]. In addition, comparing with the single lap adhesive joint (SLJ), adopting the SSJ and DSJ avoids bending when subjected to uniaxial tensile loading. However, a pure tensile loading is rare in actual applications. Commonly, the external loading is a combined loading with tension and bending together. Thus, the failure mechanism (including joint load-bearing capacity and damage level) of the joint under combined loadings should be examined deeply to promote practical applications.

Failure of adhesive joints is dictated by the mechanical properties of the adhesive [7-11] and the stress states of the adhesive layer controlled by the geometrical configurations and constraint effects $[4,12]$. In addition, failure was demonstrated to take place progressively as energy dissipates gradually at the crack tip [4,7$11,13]$. Furthermore, failure generally occurs in the adhesive layer with a lower stiffness than that of the adherends, which has been proved by previous investigations $[4,8-11,14-15]$. The onset of damage can be predicted without requiring any initial crack using the existing stress- or strain-based criteria [13]. However, the obvious disadvantage of these methods is the mesh dependence 
caused by the singularities at the edge of the adhesive. Fracture mechanics approach is mesh independent but an initial crack is indispensable [16,17]. Owing to the complex failure behavior of the adhesive joint, it is difficult to obtain a universal failure criterion to various situations. Alternatively, cohesive zone models (CZM) can simulate the damage onset and growth with mesh independence and dispensable initial crack, maintaining the possibility to characterize the behavior of the structure up to failure [7-15]. Especially, an efficient finite element computational method with the core of CZM was certified by Castagnetti et al. [18], which showed that the socalled Tied Mesh method has the advantage of numerical precision and computational speed. Moreover, the evaluation of the cohesive parameters influence was carried out by Campilho et al. [19], which allowed a critical perception of the effect of these parameters on numerical predictions.

For the mixed-mode strength, Spaggiari et al. [20] discussed various criteria aimed to thin adhesive films. In their study, they mentioned that the responses in Mode I (normal stresses) and Mode II (shear stresses) of the adhesive are significantly different. In addition, they concluded that it is difficult to find a limit stress using the traditional criteria for ductile and brittle materials. Furthermore, they also pointed out that the Stassi D'Alia criterion can find an equivalent stress value, which is valid irrespective of the loading conditions.

In the present study, the load-bearing capacity and damage level of a DSJ with various scarf angles and adhesives subjected to the combination of tension and bending are examined using a mixedmode CZM with a bilinear shape coupled with a finite element subroutine (performed in ABAQUS ${ }^{\circledR}$ [21]), which takes into account the normal-shear mixed stress state at the scarf interface. The numerical analysis is validated with existing experimental results. The effects of scarf angle $\left(30^{\circ}, 45^{\circ}, 60^{\circ}\right)$ and adhesive properties (three types) on the load-bearing capacity, the von-Mises equivalent stress distributions at the interface and damage level corresponding to the ultimate loading of DSJs are evaluated. Finally, the energy required for the joint failure, which is described as the stretch energy of the resultant loading that is equal to the area under the loaddisplacement curve of the DSJ, is also estimated.

\section{Numerical analysis}

\subsection{DSJ model}

A finite element model of a DSJ subjected to a combined loading of tension and bending is introduced for analysis, as shown in Fig. 1. Two adherends [I] with the same materials are bonded with the adhesive layer at the scarf interface. Young's

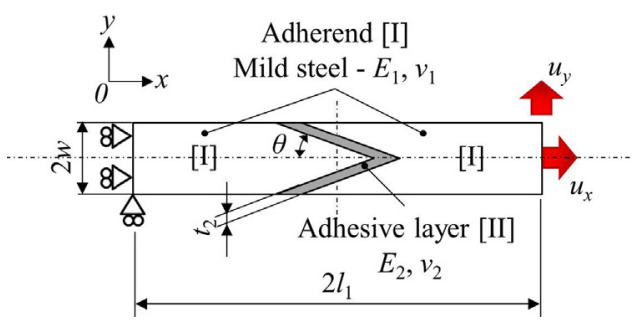

Fig. 1. A model of DSJ with boundary conditions.

Table 1

Material and geometric parameters of the DSJ.

\begin{tabular}{lllll}
\hline$E_{1}(\mathrm{GPa})[5,6]$ & $\nu_{1}[5,6]$ & $2 l_{1}(\mathrm{~mm})$ & $2 w(\mathrm{~mm})$ & $t_{2}(\mathrm{~mm})$ \\
\hline 209 & 0.29 & 100 & 20 & 0.1 \\
\hline
\end{tabular}

modulus and Poisson's ratio of the adherends [I] are denoted as $E_{1}$ and $\nu_{1}$, those of the adhesive layer [II] are $E_{2}$ and $\nu_{2}$, respectively. The length and the width of the adherends are $2 l_{1}$ and $2 w$. The thickness of the adhesive layer [II] is denoted as $t_{2}$. The material and geometric parameters are listed in Table 1 .

Supposing that the width of the adherends of the joint selected in the present study is far larger than the thickness (thin plate specimen), the DSJ can be simplified as a 2D plane-strain problem. Correspondingly, Cartesian coordinates $(x, y)$ are adopted in modeling. As for the boundary conditions, they are defined as: (1) the free end of the left adherend [I] is constrained both in the $x$ - and $y$-direction; (2) the tension and bending loading, which is simulated by controlling the displacement increment method along the $x-\left(u_{x}\right)$ and $y$-direction $\left(u_{y}\right)\left(u_{x}=2 u_{y}\right)$, is applied to the free end of the right adherend [I], respectively.

The progressive nonlinear failure occurs at the adhesive interface, which results from the extremely great difference in stiffness between the adherend and the adhesive [3]. Subsequently, a geometrical and material nonlinear numerical analysis is performed in ABAQUS ${ }^{\circledR}$ to simulate the mechanical behavior of the DSJ by adopting a CZM to simulate damage initiation and growth, which is discussed in details in Section 2.2. The parameters of the cohesive elements are set as described in Section 2.3 according to the chosen adhesive.

The FEM model with mesh details is shown in Fig. 2, where the geometrical thickness of the adhesive layer (for easier visual effect) is different from the real thickness $t_{2}$. Accordingly, the adhesive layer [II] is built as a single layer using four-node cohesive elements, which share nodes with the neighboring elements in the adherends (as shown in Fig. 2 with a magnified view of the CZM elements at the interface and the connection details). The adherends [I], which are high-strength steel [5,6], are defined as isotropic elastic for simplicity. In addition, they are meshed using four-node quadrilateral plane-strain elements. The adhesive region is densely meshed using biasing effects while sparse meshes are used in other regions for higher computational accuracy. In addition, optional viscous damping is implemented between node pairs to improve convergence [15].

In order to examine the effects of the scarf angle $\theta$ on the performance of the joint, it is chosen as $30^{\circ}, 45^{\circ}$ and $60^{\circ}$, respectively. In addition, the effects of the properties of adhesives on the performances of the joint are also analyzed, in which three adhesives [8] are selected: a brittle adhesive (AV138/HV998) [22], an intermediate adhesive (Hysol EA 9321) [23] and a ductile adhesive (Hysol EA 9361) [24], respectively. The tensile stressstrain curves of the bulk adhesives are shown in Fig. 3 [8].

\section{2. $C Z M$}

Based on the Traction-Separation (T-S) law, CZM is widely used to analyze the de-cohesion in composite structures [8-11,13-15]. It must be pointed out that the adhesive layer using CZM is a generalized interface phase rather than a material. $T-S$ curve can be considered as a representation of the constitutive relation of the equivalent interface [9]. A bilinear assumption model [25-27], in which a critical energy release rate $G_{c}$ and a cohesive strength $\sigma_{u}$ are vital to capture the interface separation behavior [9-11,15,28], is employed in this study. Owing to the combined loadings, a complex stress state of the joint is present with mixed-mode (Mode I and II) damage propagation, as shown in Fig. 4 [8-11,13,19].

According to the existing research findings [8-11,13], it can be noticed that the constitutive relationship before damage onset is calculated using:

$\boldsymbol{\sigma}=D \boldsymbol{\delta}$

where $\boldsymbol{\sigma}, \boldsymbol{\delta}$ and $\mathbf{D}$ are the vector of interface finite element stresses, the vector of relative displacements and a diagonal matrix 


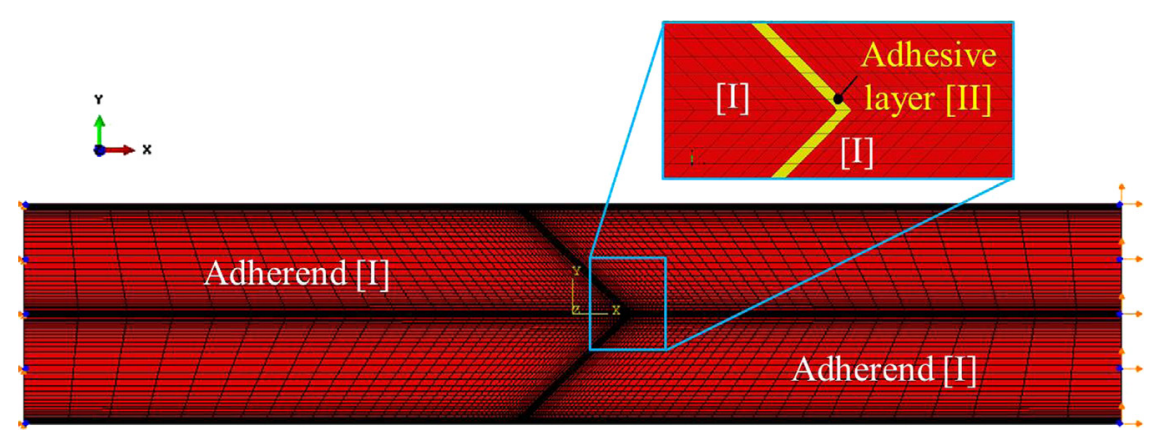

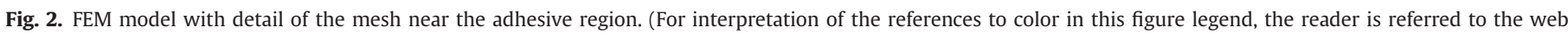
version of this article.)

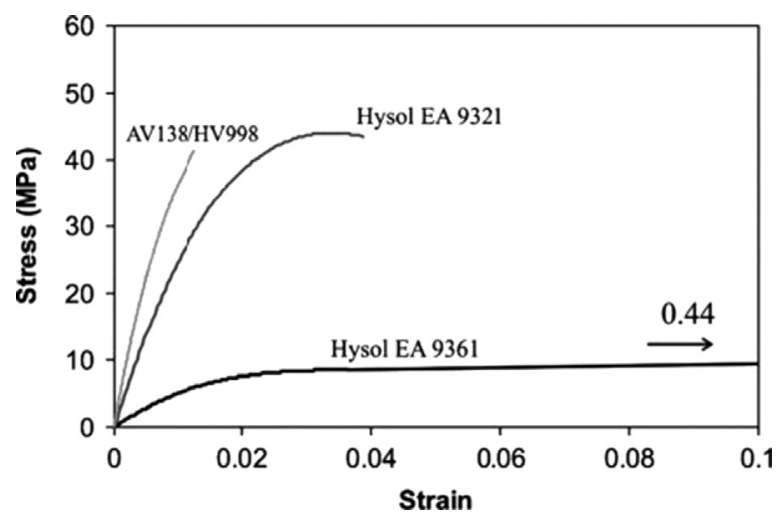

Fig. 3. Tensile stress-strain curves of the various adhesives tested [8].

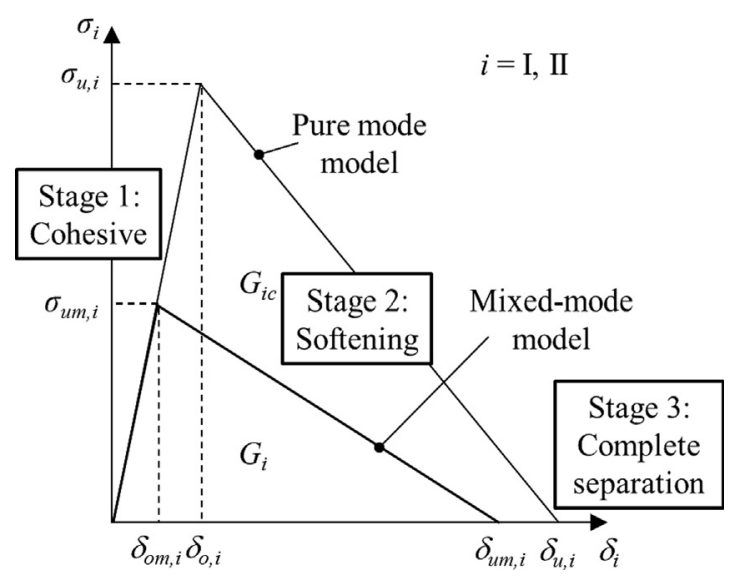

Fig. 4. Pure and mixed-modes damage models [8].

containing the interface stiffness, respectively. For each pure mode, the material softens progressively with damage after $\delta_{o, i}$ $\left(\sigma_{u, i}\right)$. With the damage propagation, the stress in the triangle decreases. The softening relationship is described as:

$\boldsymbol{\sigma}=(\mathbf{I}-\mathbf{E}) D \boldsymbol{\delta}$

where $\mathbf{I}$ is the identity matrix and $\mathbf{E}$ a diagonal matrix containing the damage parameters defined by:

$e_{i}=\frac{\delta_{u, i}\left(\delta_{i}-\delta_{o, i}\right)}{\delta_{i}\left(\delta_{u, i}-\delta_{0, i}\right)}$

where $\delta_{i}$ is the current relative displacement in mode $i$ and $\delta_{o, i}$ the displacement corresponding to the onset of damage. The maximum relative displacement $\delta_{u, i}$ of complete separation is obtained by equating the area under the soften curve with the critical energy release rate:

$G_{i c}=1 / 2 \sigma_{u, i} \delta_{u, i}$

Sancaktar [29] discussed the failure criteria under monotonic loading conditions systematically. In his work, a parabolic failure condition and an elliptical one were provided, which represent the upper limit for catastrophic threshold values under cyclic loading. Under the mixed-mode conditions, damage initiation is controlled using a quadratic stress criterion as following $[8,9,13,19]$.

$\left(\sigma_{\mathrm{I}} / \sigma_{u, \mathrm{I}}\right)^{2}+\left(\sigma_{\mathrm{II}} / \sigma_{u, \mathrm{II}}\right)^{2}=1$

which assumes that a pure compressive deformation or stress state does not initiate damage. When Eq. (5) is satisfied, the damage level is expressed utilizing a single damage variable $D$ according to the total displacement jump $\Delta\left(\Delta=\sqrt{\left(\delta_{\mathrm{I}}\right)^{2}+\left(\delta_{\mathrm{II}}\right)^{2}}\right)$ as follows [9-11]:

$D=\frac{\Delta_{f}\left(\Delta_{\max }-\Delta_{o}\right)}{\Delta_{\max }\left(\Delta_{f}-\Delta_{o}\right)}$

where $\Delta_{o}$ and $\Delta_{f}$ are the total displacement of the damage onset and complete failure; $\Delta_{\max }$ represents the maximum total displacement ever experienced during the loading history. $\Delta_{f}$ is calculated by $\Delta_{f}=2 G / \sqrt{\left(\delta_{0, \mathrm{I}}\right)^{2}+\left(\delta_{0, \mathrm{II}}\right)^{2}}$, where $G$ is the total energy released during the separation of the adhesive layer.

It is assumed that a linear fracture criterion determines the damage propagation, which is expressed as $[8,9,13,19]$ :

$G_{\mathrm{I}} / G_{\mathrm{IC}}+G_{\mathrm{II}} / G_{\text {IIC }}=1$

The critical energy release rate in each mode of complete failure is described using the area of the triangle in Fig. 4:

$G_{i}=1 / 2 \sigma_{u m, i} \delta_{u m, i}$

The relative displacements for each mode corresponding to damage onset $\delta_{o m, i}$ and ultimate failure $\delta_{u m, i}$ can be expressed as:

$\delta_{\text {om }, i}=\frac{\beta_{i} \delta_{\mathrm{om}}}{\sqrt{1+\beta_{\mathrm{II}}^{2}}}$

$\delta_{u m, i}=\frac{\beta_{i} \delta_{u m}}{\sqrt{1+\beta_{\mathrm{II}}^{2}}}$

where $\beta_{i}$ is the mode ratio $\left(\beta_{i}=\delta_{i} / \delta_{\mathrm{I}}\right) ; \delta_{\text {om }}, \delta_{u m}$ are the equivalent mixed-mode relative displacements, which are given as follows:

$\delta_{o m}=\delta_{o, \mathrm{I}} \delta_{o, \mathrm{II}} \sqrt{\frac{1+\beta_{\mathrm{II}}^{2}}{\delta_{o, \mathrm{II}}^{2}+\beta_{\mathrm{II}}^{2} \delta_{o, \mathrm{I}}^{2}}}$

$\delta_{u m}=\frac{1+\beta_{\mathrm{II}}^{2}}{\delta_{o m}\left(\frac{1}{\delta_{0,1} \delta_{u, 1}}+\frac{\beta_{\mathrm{II}}^{2}}{\delta_{0, \mathrm{II}} \delta_{u, \mathrm{II}}}\right)}$ 
The damage parameters can be obtained by substituting Eqs. (11) and (12) to Eq. (3).

\subsection{Cohesive parameters}

A parameterized bilinear $T-S$ law for mixed-mode is used to model de-cohesion of DSJ. The thickness of the adhesive layer $t_{2}$ is introduced to the stiffness matrix $\mathbf{D}$, which is calculated from the ratio between the elastic modulus of the material and the thickness of the adhesive layer. To fully characterize the cohesive law for each pure mode (I and II), critical energy release rate $G_{i c}$ and cohesive strength $\sigma_{u, i}(i=\mathrm{I}$, II) should be defined in advance $[13,15]$. In order to examine the effects of the adhesive types on the performances of DSJ, three adhesives (brittle, intermediate and ductile) are selected.

The constitutive parameters of the employed adhesives are listed in Table 2. The adhesive with definite thickness would dissipate energy $G_{p}$ result from the plastic deformation, which is approximately estimated as $t_{2} A_{c}[9-11,30]$. Thus, the plastic dissipation energy is considered as the mean plastic work per unit adhesive volume, where $A_{c}$ is the area below the stress-strain curve of the adhesive material [9-11]. Therefore, the total fracture energy $G$ includes the intrinsic energy for adhesive layer separation and plastic dissipation energy. For the effect of the adhesive thickness, Castagnetti et al. discussed and then concluded that the intrinsic static strength of the adhesive increases significantly as the adhesive thickness decreases when cohesive failure occurs [31]. In the present study, the cohesive strength in each mode is considered to be equal to the yield strength of the adhesive $\left(\sigma_{u, \mathrm{I}}=\sigma_{u, \mathrm{II}}=\sigma_{y a}\right)$ for simplicity $[8,16,32-34]$.

\section{Results and discussion}

\subsection{Numerical results}

\subsubsection{Load-bearing capacity}

The load-bearing capacity of the adhesive joints has been extensively estimated employing the ultimate loading $\left(F_{u}\right)$ [8-11,32-34,35]. It is assumed that the loading $F$ of DSJ is combined with the tensile loadings in the $x$ - and $y$-directions $\left(F=\sqrt{\left(F_{x}\right)^{2}+\left(F_{y}\right)^{2}}\right)$. The displacement $u$ is defined as $u=\sqrt{\left(u_{x}\right)^{2}+\left(u_{y}\right)^{2}}$.

Fig. 5 shows the variations of the resultant loading $F$ as a function of the displacement $u$ of DSJs with a series of scarf angles and adhesive types, where $F$ is the resultant force per unit thickness of the joint. It can be observed that the load-bearing capacity of the DSJ increases as the scarf angle $\theta$ decreases, regardless of the adhesive types. In addition, for a given scarf angle $\theta$, as shown in Figs. 5 and 6, the load-bearing capacity of the DSJ with the brittle adhesive is significantly larger than that of the joint with the ductile one. On the contrary, as shown in Fig. 5 for

Table 2

Adhesives constitutive parameters [8].

\begin{tabular}{lccl}
\hline Parameters & $\begin{array}{l}\text { AV138/ } \\
\text { HV998 } \\
\text { (brittle) }\end{array}$ & $\begin{array}{l}\text { Hysol EA 9321 } \\
\text { (intermediate) }\end{array}$ & $\begin{array}{l}\text { Hysol EA } \\
9361 \\
\text { (ductile) }\end{array}$ \\
\hline Young's modulus E $(\mathrm{GPa})$ & 4.59 & 3.87 & 0.67 \\
Poisson's ratio $\nu$ & 0.35 & 0.36 & 0.4 \\
Yield strength $\sigma_{y a}(\mathrm{MPa})$ & 36.49 & 21.99 & 4.23 \\
$\begin{array}{c}\text { Critical energy release rate } \\
\quad \text { (Mode I) } G_{\text {Ic }}(\mathrm{N} / \mathrm{mm})\end{array}$ & 0.3 & 0.45 & 2.61 \\
$\begin{array}{c}\text { Critical energy release rate } \\
\text { (Mode II) } G_{\text {IIc }}(\mathrm{N} / \mathrm{mm})\end{array}$ & 0.6 & 0.9 & 5.22 \\
\hline
\end{tabular}

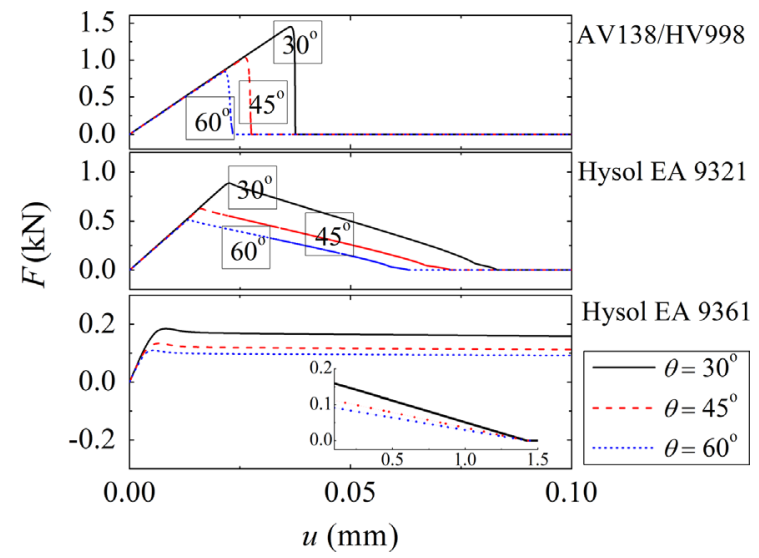

Fig. 5. Load $(F)$-displacement $(u)$ curves of DSJs with a series of scarf angles $\theta$ and adhesives under combined loadings $\left(t_{2}=0.1 \mathrm{~mm}\right)$.

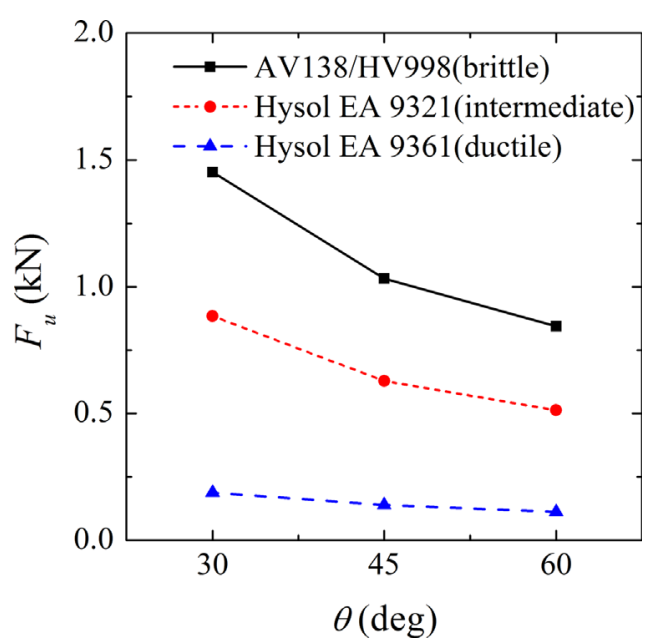

Fig. 6. Variation of the ultimate loading $F_{u}$ with respect to the scarf angle $\theta$.

the joint with the brittle adhesive, the ultimate applied displacement $u_{u}$ until complete failure is quite smaller than that of the joint with the ductile one.

\subsubsection{Stress distributions in the adhesive layer}

In this section, von-Mises equivalent stress $\sigma_{\text {eqv }}$ corresponding to reaching the ultimate loading $F_{u}$ is selected to examine the stress distributions. The path for evaluating the stress distribution is chosen as the middle line of the equivalent interface geometrically. Fig. 7 shows the distributions of the von-Mises equivalent stress $\sigma_{\text {eqv }}$ in the adhesive layer, where the abscissa is the normalized position $(y / w)$ along the width direction. It can be found that the von-Mises equivalent stress $\sigma_{\text {eqv }}$ at the upper half of the bonded area (from $y / w=1.0$ to $y / w=0.0$ ) is lower than that at the lower half (from $y / w=0.0$ to $y / w=-1.0$ ). Moreover, it can also be observed that the stress level at the bonding interface of DSJs with the brittle adhesive is higher than that of DSJs with the ductile one. Furthermore, the von-Mises equivalent stress $\sigma_{\text {eqv }}$ increases as the scarf angle $\theta$ decreases. For the effect of the scarf angel on the stress distributions, Sancaktar and Narayan [36] reported that the distributions of stresses become more uniform along the adhesive layer as the scarf angle increases. In addition, they also obtained the reverse trends of the transverse/shear stress and normal stress distributions with respect to the scarf angle.

\subsubsection{Damage level of the adhesive layer}

Damage is a progressive process of crack initiation and propagation, which takes place in the adhesive layer. Correspondingly, 

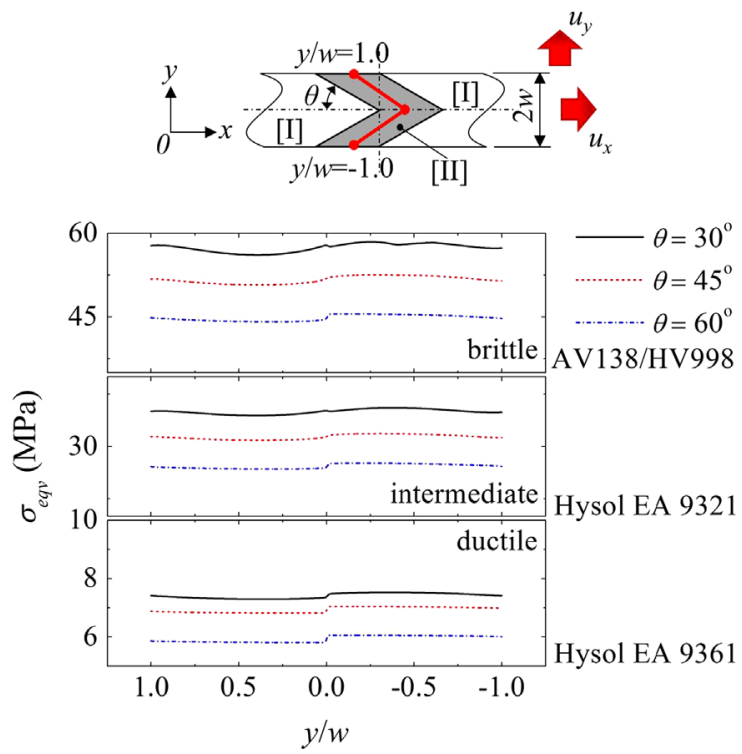

Fig. 7. Von-Mises equivalent stress $\sigma_{\text {eqv }}$ distributions along the middle line of the adhesive layer of DSJs corresponding to the ultimate loading.
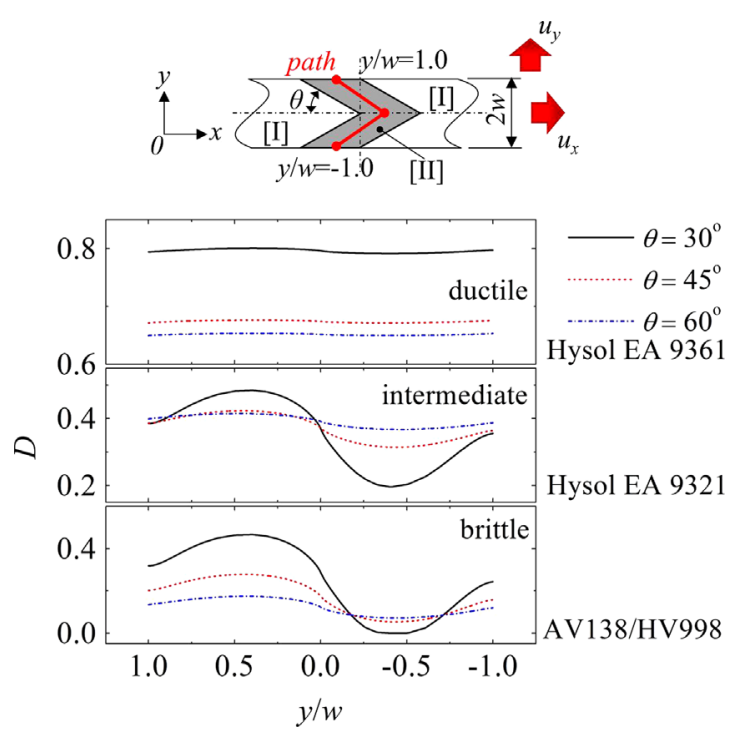

Fig. 8. Damage distributions at the interface of DSJ corresponding to the ultimate loading.

as described in Eq. (6), the damage variable $D$ increases monotonically from 0 (corresponding to damage initiation) to 1 (corresponding to complete failure). Fig. 8 shows the damage distributions along the schematic path corresponding to the ultimate loading $F_{u}$. The abscissa represents the position of the interface using the normalized expression $y / w$ along the width direction. From Fig. 8, comparing the lower half of the bonding area (from $y / w=0.0$ to $y / w=-1.0$ ), the damage level at the upper half (from $y / w=0.0$ to $y / w=1.0$ ) is higher. In addition, the damage level increases as the scarf angel $\theta$ decreases. Furthermore, it can also be observed that with the increase of the ductility of the adhesive, the damage level increases and the differences between the two halves of the bonding area shrink gradually.

\subsection{Discussion}

In the typical loading evolution process, the applied loading experiences the ascending period until reaching the ultimate value and a descending period in sequence [9-11], which is also seen in Fig. 5. Correspondingly, the ultimate loading is chosen to assess the load-bearing capacity of the joint. In the simulated model, CZM is selected to describe the separation behavior of the adhesive joint controlled by cohesive strength and critical energy release rate [9-11].

As listed in Table 2, it can be seen that the cohesive strength (assumed to be equal to the bulk yield strength) of the adhesive decreases as the critical energy release rate increases. Subsequently, for the joint with the brittle adhesive (AV138/HV998), the ultimate loading $F_{u}$, which is governed by the cohesive strength of the chosen adhesive, is larger than those of the joints with the intermediate (Hysol EA 9321) and ductile ones (Hysol EA 9361) (as shown in Figs. 5 and 6).

As shown in Fig. 5, the resultant loading $F$ declines after reaching the ultimate value at each given scarf angle $\theta$ owing to the damage propagation in the adhesive layer. However, the downward slopes are different for the joints with different adhesives. For the joint with the brittle adhesive AV138/HV998, the resultant loading drops to zero sharply showing significant brittle property. For the joint with the intermediate adhesive Hysol EA 9321, the downward slope of the resultant loading becomes smaller. For the joint with the ductile adhesive Hysol EA 9361, the resultant loading drops slowly until zero experiencing a quite large displacement, which illustrates a significant ductile property. It is assumed that the different downward trends are decided by the critical energy release rate of the adopted adhesive [8-11,32-34]. Neglecting the influences of the plastic dissipation energy, the ascending Sort of the critical energy release rate for the three adhesives is the brittle, the intermediate and the ductile adhesives (as shown in Table 2).

For the joint with the brittle adhesive, with the increase of the resultant loading, it can be considered that the failure of the adhesive layer occurs without plastic deformation. However, for the ductile adhesive, the strain variation sensitivity of stress is low after reaching the maximum stress, which indicates the constitutive property as shown in Fig. 3 [8]. As shown in Fig. 5, the loading drops so slow for the joints with the intermediate and ductile adhesives that the complete separations occur at quite large displacements comparing with the joint with the brittle adhesive. It can be assumed that more comprehensive estimation of the joint performance is required except the load-bearing capacity only.

As shown in Fig. 8, the damage level for the joint with the ductile adhesive corresponding to the ultimate loading is higher and more uniform than those of the joints with the intermediate and brittle ones, which indicates that the joint with the ductile adhesive has the ability to distribute the loading over a large cohesive zone with a more uniform distribution. This confirms the results from the other research [8-11,37]. In addition, with the critical energy release rate of the selected adhesive decreasing, the difference between the upper half and lower half of the interface along the schematic path increases because the uniformity of the loading distributions decreases. Owing to the combined loadings of tension and bending, the lower half of the cohesive zone is subjected to compressive loading in the $y$-direction. As a result, the damage level of the lower half of the adhesive layer is lower than that of the upper half in the cohesive zone.

Returning to Fig. 7, the stress level of the lower half of the interface is higher than that of the upper half for each scarf angle and adhesive. By contrast, as shown in Fig. 8, owing to the compressive loading applied on the lower half of the interface, the damage level of the lower half of the interface becomes lower. It is the result from the failure initiation criterion shown in Eq. (5), which assumes that a compressive stress does not initiate damage. Actually, the failure of the joint is dictated by the damage evolution. 
As mentioned above, owing to the properties varying with different adhesives, a more reasonable evaluation method of the adhesive joint performance is desired. In the present study, the failure energy, which is described as the work done by the resultant loading with respect to the displacement until complete failure, is employed to estimate the joint performance:

$E_{f}=\int_{0}^{u_{u}} F d u$

where $u_{u}$ is the ultimate displacement corresponding to the resultant loading $F$ dropping from the ultimate value to zero, which indicates complete failure. The physical significance of the failure energy is the energy required for the failure of the joint with a given scarf angle and selected adhesive. Fig. 9 shows the comparison of the failure energy $E_{f}$ among DSJs with the brittle, intermediate and ductile adhesives under combined loadings. It can be observed that the energy required for the failure of the DSJ with the brittle adhesive is smaller than that of the DSJ with the intermediate and ductile ones. Furthermore, it can be concluded that the adhesive joint performance is controlled both by the ultimate loading and by the ultimate displacement until complete failure.

\subsection{Validation of the present simulated method}

In order to verify the present method, the numerical results are compared with the results from the existing experimental measurements [3], which focus on the variation trend with respect to the scarf angel. The variation of the normalized ultimate loading $F_{u} / 2 w t \sigma_{y a}$ with the scarf angle $\theta$ is shown in Fig. 10 . The DSJs were examined subjected to uniaxial tensile loading along the $x$-direction both in the work performed by Gacoin et al. [3] and the following calculations using the present method.

In the experiments carried by Gacoin et al. [3], an epoxy resin SIKADUR $30 \operatorname{COLLE}^{\circledR}$ (SIKA, Paris, France) was adopted to DSJs with the scarf angle $\theta 6^{\circ}, 18^{\circ}$ and $33^{\circ}$, respectively. In addition, the width $2 w$ and the thickness $t$ of DSJ were both chosen as $10 \mathrm{~mm}$. Moreover, the yield stress $\sigma_{y a}$ of the adopted adhesive was $24 \mathrm{MPa}$. On the other hand, in order to compare the ultimate loading variation in the same range of the scarf angel, the scarf angle $\theta$ is set as $10^{\circ}, 15^{\circ}, 18^{\circ}, 20^{\circ}, 25^{\circ}, 30^{\circ}$ and $33^{\circ}$, respectively in the present study. For the plane-strain problem in the present study, the unit thickness of the joint can be considered as 1 .

It can be found that the load-bearing capacity of the DSJ decreases as the scarf angle $\theta$ increases, which shows a good agreement between the variation trends of the curves with respect

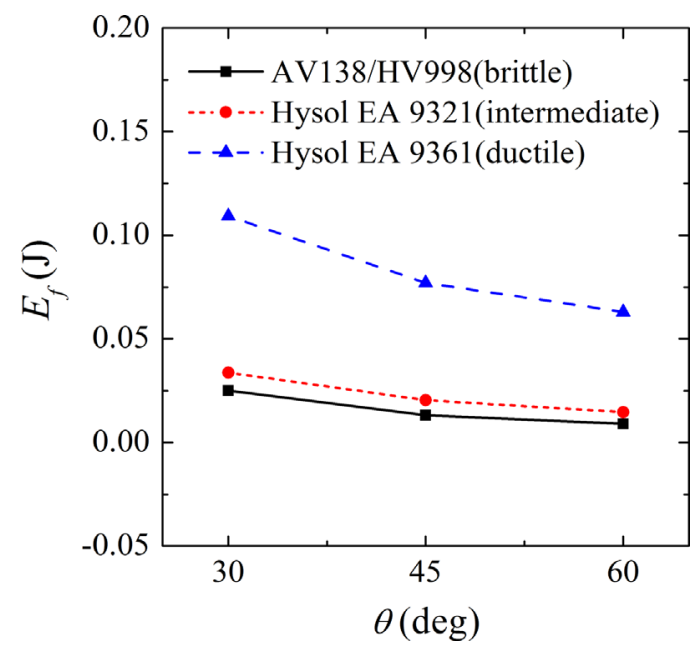

Fig. 9. Variation of the failure energy $E_{f}$ with the scarf angle $\theta$.

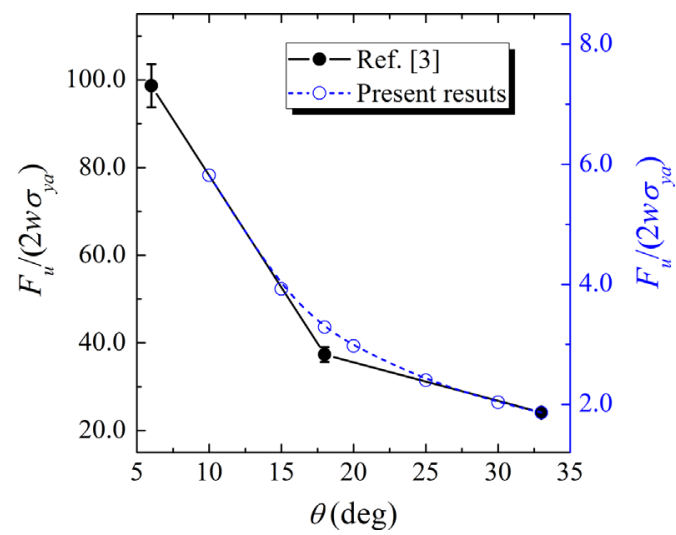

Fig. 10. Comparisons of the normalized ultimate loading vs. scarf angle between the present results and existing experimental measurements [3] of DSJs under uniaxial tensile loading.

to the scarf angle $\theta$. Similarly, it can be concluded that the present simulated method is effective in analyzing the failure mechanism of DSJs with various scarf angles and adhesives subjected to tensile loadings and combined loadings.

\section{Conclusions}

In the present study, the load-bearing capacity and the damage level of DSJs with a series of angles and adhesives subjected to a combined loading of tension and bending are numerically investigated. A mixed-mode CZM with a bilinear shape is used. The following are specific conclusions.

(1) Controlling by the bulk cohesive strength, the ultimate loading of the DSJ with the brittle adhesive is higher than that of the joint with the ductile one. On the other hand, the applied displacement until complete failure of the DSJ with the brittle adhesive is quite smaller than that of the joint with the ductile one.

(2) The von-Mises equivalent stress $\sigma_{\text {eqv }}$ at the upper half of the cohesive zone of the DSJ is larger because of the effects of the compressive loading. In addition, the stress level of the DSJ decreases when the adhesive varies from brittle to ductile.

(3) Comparing with the lower half of the bonded area, the damage level at the upper half of the adhesive layer is higher. Moreover, the difference of the damage levels between the two halves becomes weaker as the ductility of the selected adhesive increases. Since the ductile adhesive has the ability to distribute the loading over a large scale effectively, the interface damage level of the DSJ increases with the ductility of the employed adhesive.

(4) The failure of the DSJ is governed by the ultimate loading and by the displacement until complete failure. Considering the limitation of the traditional criteria, the failure energy, which is defined as the integral of the loading with respect with the displacement of DSJ, is adopted to measure the joint performance, which shows that the energy required for the failure of DSJs with the ductile adhesive is higher than that of the joint with the brittle one.

(5) The variations of the ultimate loading, the von-Mises equivalent stress and interface damage level corresponding to the ultimate loading, and the failure energy of the DSJ with a given adhesive with respect to the scarf angle were discussed, which are inversely proportional.

(6) The present numerical method is validated with existing experimental results. 


\section{Acknowledgements}

Support from the National Natural Science Foundation of China, Grant No. 11202222 is gratefully acknowledged.

\section{References}

[1] Kimiaeifar A, Toft H, Lund E, Thomsen OT, Sørensen JD. Reliability analysis of adhesive bonded scarf joints. Eng Struct 2012;35:281-7.

[2] Adin Hamit. The investigation of the effect of angle on the failure load and strength of scarf lap joints. Int J Mech Sci 2012;61:24-31.

[3] Gacoin A, Lestriez P, Assih J, Objois A, Delmas Y. Comparison between experimental and numerical study of the adhesively bonded scarf joint and double scarf joint: influence of internal singularity created by geometry of the double scarf joint on the damage evolution. Int J Adhes Adhes 2009;29:572-9.

[4] Afendi Mohd, Teramoto Tokuo, Bakri Hairul Bin. Strength prediction of epoxy adhesively bonded scarf joints of dissimilar adherends. Int J Adhes Adhes 2011;31:402-11.

[5] He D, Sawa T, Iwamoto T, Hirayama Y. Stress analysis and strength evaluation of scarf adhesive joints subjected to static tensile loadings. Int J Adhes Adhes 2010;30:387-92.

[6] He D, Sawa T, Karami A. Stress analysis and strength evaluation of scarf adhesive joints with dissimilar adherends subjected to static tensile loadings. JSME Solid Mater 2009;3:1033-44.

[7] Adams RD, Wake WC, Comyn J. Structural adhesive joints in engineering. Chapman and Hall; 1997.

[8] da Silva LFM, Rodrigues TNSS, Figueiredo MAV, de Moura MFSF, Chousal JAG. Effect of adhesive type and thickness on the lap shear strength. J Adhes 2006;82:1091-115.

[9] Xu W, Wei Y. Influence of adhesive thickness on local interface fracture and overall strength of metallic adhesive bonding structures. Int J Adhes Adhes 2013;40:158-67.

[10] Xu W, Wei Y. Assessments for impact of adhesive properties: modeling strength of metallic single lap joint. J Adhes Sci Technol 2013;27:9-29.

[11] Xu W, Wei Y. Strength and interface failure mechanism of adhesive joints. Int Adhes Adhes 2012;34:80-92.

[12] Pardoen T, Ferracin T, Landis CM, Delannay F. Constraint effects in adhesive joint fracture. Int J Mech Phys Solids 2005;53:1951-83.

[13] Campilho RDSG, de Moura MFSF, Ramantani DA, Morais JJL, Domingues JJMS Tensile behavior of three-dimensional carbon-epoxy adhesively bonded single- and double-strap repairs. Int J Adhes Adhes 2009;29:678-86.

[14] Rudawska Anna. Adhesive joint strength of hybrid assemblies: titanium sheetcomposites and aluminium sheet-composites-experimental and numerical verification. Int J Adhes Adhes 2010;30:574-82.

[15] Peter Gustafson A, Anthony Waas M. The influence of adhesive constitutive parameters in cohesive zone finite element models of adhesively bonded joints. Int J Solids Struct 2009;46:2201-15.

[16] de Moura MFSF, Goncalves JPM, Chousal JAG, Campilho RDSG. Cohesive and continuum mixed-mode damage models applied to the simulation of the mechanical behaviour of bonded joints. Int J Adhes Adhes 2008;28:419-26.
[17] Ridha M, Tan VBC, Tay TE. Traction-separation laws for progressive failure of bonded scarf repair of composite panel. Compos Struct 2011:93:1239-45.

[18] Castagnetti D, Dragoni E, Spaggiari A. Failure analysis of complex bonded structures: experimental tests and efficient finite element modeling by tied mesh method. Int J Adhes Adhes 2011;31:338-46.

[19] Campilho RDSG, Banea MD, Neto JABP, da Silva LFM. Modelling of single-lap joints using cohesive zone models: effect of the cohesive parameters on the output of the simulations. J Adhes 2012;88:513-33.

[20] Spaggiari A, Castagnetti D, Dragoni E. Mixed-mode strength of thin adhesive films: experimental characterization through a tubular specimen with reduced edge effect. J Adhes 2013;89:660-75.

[21] Abaqus ${ }^{\mathbb{R}} 6.10$ Analysis User's Manual, volume IV: elements, (C) Dassault Systemes; 2010.

[22] Araldite ${ }^{\mathbb{B}}$ AV 138M with Hardener HV 998 technical data sheet; May 2004.

[23] Hysol ${ }^{\mathbb{R}}$ EA9321 data sheet, Henkel Corporation, Aerospace Group, 2850 Willow Pass Road, PO Box 312, Bay Point, CA 94565 USA, 925.458.8000.

[24] Hysol $^{\mathbb{R}}$ EA9361 data sheet, Henkel Corporation, Aerospace Group, 2850 Willow Pass Road, PO Box 312, Bay Point, CA 94565 USA, 925.458.8000.

[25] Li H, Chandra N. Analysis of crack growth and crack-tip plasticity in ductile materials using cohesive zone models. Int J Plast 2003;19:849-82.

[26] Chandra N, Li H, Shet C, Ghonem H. Some issues in the application of cohesive zone models for metal-ceramic interfaces. Int J Solids Struct 2002;39:2827-55.

[27] Ghosh S, Ling Y, Majumdar B, Kim R. Interfacial debonding analysis in multiple fiber reinforced composites. Mech Mater 2000;32:561-91.

[28] Sørensen BF, Kirkegaard P. Determination of mixed mode cohesive laws. Eng Fract Mech 2006;73:2642-61.

[29] Sancaktar Erol. Fracture aspects of adhesive joints: material, fatigue, interphase, and stress concentration considerations. J Adhes Sci Technol 1995;9:119-47.

[30] Wei YG, Zhao HF. Peeling experiments of ductile thin films along ceramic substrates-critical assessment of analytical models. Int J Solids Struct 2008:45:3779-92.

[31] Castagnetti D, Spaggiari A, Dragoni E. Effect of bondline thickness on the static strength of structural adhesives under nearly-homogeneous shear stresses. J Adhes 2011;87:780-803.

[32] da Silva LFM, Carbas RJC, Gritchlow GW, Figueiredo MAV, Brown K. Effect of material, geometry, surface treatment and environment on the shear strength of single lap joints. Int J Adhes Adhes 2009:29:621-32.

[33] Banea MD, da Silva LFM. Mechanical characterization of flexible adhesives. J Adhes 2009;85:261-85.

[34] da Silva LFM, Gritchlow GW, Figueiredo MAV. Parametric study of adhesively bonded joints. J Adhes Sci Technol 2008;22:1477-94.

[35] De Morais AB, Pereira AB, Teixeira JP, Cavaleiro NC. Strength of epoxy adhesive-bonded stainless-steel joints. Int J Adhes Adhes 2007;27:679-86.

[36] Sancaktar Erol, Narayan Kiran. Substrate volume and stress gradient concepts in mechanical adhesion: analysis of single straight sections. J Adhes Sci Technol 2012;13:237-71.

[37] Loureiro AL, da Silva LFM, Sato C, Figueiredo MAV. Comparison of the mechanical behavior between stiff and flexible adhesive joints for the automotive industry. J Adhes 2010;86:765-87. 